\title{
A comparison between two types of indwelling pleural catheters for management of malignant pleural effusions
}

\author{
Sushilkumar Satish Gupta ${ }^{1}$, Charalampos S. Floudas ${ }^{2}$, Abhinav B. Chandra ${ }^{3}$ \\ ${ }^{1}$ Department of Pulmonary and Critical Care Medicine, Maimonides Medical Center, Brooklyn, New York, USA; ${ }^{2}$ Department of Internal Medicine, \\ Maimonides Medical Center, Brooklyn, New York, USA; ${ }^{3}$ Department of Hematology and Oncology, Yuma Regional Medical Center Cancer \\ Center, Yuma, Arizona, USA \\ Contributions: (I) Conception and design: All authors; (II) Administrative support: All authors; (III) Provision of study materials or patients: All \\ authors; (IV) Collection and assembly of data: SS Gupta, CS Floudas; (V) Data analysis and interpretation: SS Gupta, CS Floudas; (VI) Manuscript \\ writing: All authors; (VII) Final approval of manuscript: All authors. \\ Correspondence to: Sushilkumar Satish Gupta, MD. Fellow, Department of Pulmonary and Critical Care Medicine, Maimonides Medical Center, \\ 4802,10th avenue, Brooklyn, New York, USA. Email: medsushil13@gmail.com.
}

Background: Malignant pleural effusion (MPE) is a common cause of quality of life deterioration in
patients with advanced cancer. Management options include chemical pleurodesis with a sclerosing agent
such as doxycycline or talc powder, surgery, and also the placement of tunneled indwelling pleural catheters
(IPCs). Two different IPC types are mostly used in the USA.
Methods: We conducted a single-center retrospective study with the objective to compare the efficacy
and safety profiles of two IPC systems. Patients with a diagnosis of malignancy, who received IPCs by the
interventional radiology department of our hospital from January 2013 to March 2015, were identified in the
local database and a chart review was performed to record characteristics and outcomes. Patients without a
diagnosis of malignancy or with pleural effusions of cardiac origin were excluded from the study.
Results: We identified 27 patients with a median age of 59.0 years. Eighty patients received Aspira catheter
while nine patients received PleurX catheter, and seven patients achieved spontaneous pleurodesis. The
median length of stay (LOS) was 9 days for the Aspira group (AG), as compared to 13 days for the PleurX
group (PG) (overall median LOS was 10 days; range, 2-62 days). The rate of catheter-related complications
(pain, obstruction, loculations, infection, hemorrhage) was $39 \%$ (seven patients) for the AG and $33 \%$ (three
patients) for the PG (overall ten patients, 37\%).

Conclusions: In our study, outcomes and safety were similar for patients receiving either type of IPC, Aspira or PleurX.

Keywords: PleurX; Aspira; indwelling pleural catheter (IPC); malignant pleural effusion (MPE); cancer; palliative; end of life

Submitted Jan 14, 2018. Accepted for publication May 02, 2018.

doi: $10.21037 /$ jtd.2018.05.57

View this article at: http://dx.doi.org/10.21037/jtd.2018.05.57

\section{Introduction}

Malignant pleural effusion (MPE) indicates disseminated cancer (1) with a mean survival period of 6 months depending on the type of cancer (2). Approximately 150,000 cases of MPE are diagnosed yearly in the United States (3). Lung cancer accounts for more than a third of
MPE, followed by breast cancer, lymphomas and other malignancies (4). MPEs can cause dyspnea, cough, chest discomfort, and significantly affect the quality of life of the patient (4). Currently there are various techniques available for MPE management and symptom palliation, which also remains the primary objective.

In MPE, the treatment aims at fluid removal, lung 
re-expansion, and creation of pleural space adhesions (pleurodesis) to prevent recurrence. Options for recurrent MPE include chemical pleurodesis and indwelling pleural catheters (IPCs) (5). Sclerosing agents used for chemical pleurodesis include talc powder (magnesium silicate), doxycycline, bleomycin and silver nitrate $(6,7)$. Talc pleurodesis was first introduced in 1935 by Norman Bethune; talc is introduced into the pleural cavity with tube thoracostomy (slurry) or by thoracoscopy (insufflation). Surgical pleurodesis or a decortication can be performed by using thoracotomy or video-assisted thoracoscopy surgery (VATS). IPCs were approved by the FDA in 1997 and currently there are two commonly used tunneled pleural catheter types, the PleurX catheter (Denver Biomedical, now CareFusion) and the Aspira (Bard) catheter. Both catheters are made of silicon rubber with internal diameter of $15.5 \mathrm{~F}$. For pleural fluid drainage, the PleurX catheter uses vacuum collecting bottles while the Aspira catheter has a manual pump to facilitate collection in a bag. Fluid production can decrease over time with spontaneous pleurodesis occurring eventually, and then the IPC can be removed. The use of the PleurX IPC has been shown to result in symptomatic improvement in adults and children (8), effusion control, improvement in survival $(9,10)$, and appears to be cost-effective, reducing length of stay (LOS) in comparison to pleurodesis with talc or doxycycline, and VATS $(5,6)$. At our center, both types of IPCs are placed by the interventional radiology (IR) department. The use of the Aspira catheter has not been studied in the literature in comparison to either talc powder or the PleurX catheter, as shown by a search of the literature available in PubMed on September 2017. While the two IPC types share the similar underlying principle, there is a difference in the way the fluid removal is facilitated, in one case with a vacuum bag (PleurX), in the other with a manual pump (Aspira). There has been no comparison between the two IPC types either, therefore we conducted an investigation of the efficacy and the outcomes of both IPC types for the management of MPEs. We conducted a single-center retrospective study with the objective to compare the efficacy and safety profiles of these two IPC systems.

\section{Methods}

The study was approved by the Institutional Review Board of our institution, Maimonides Medical Center. Patients that received IPCs by the IR department of our hospital from January 2013 to March 2015 were identified in the local database and a retrospective chart review was performed to record patient characteristics and outcomes. Both IPC types are used interchangeably in our IR, without specific selection criteria. We looked for non-pediatric patients, older than 21 years of age, admitted to our center with a diagnosis of cancer and pleural effusion. Patients without an existing histologic diagnosis of malignancy or with pleural effusions found to be of cardiac origin were excluded from the study. Patients under the age of 21 years were excluded from the study. We recorded age, sex, type of cancer, Eastern Cooperative Oncology Group (ECOG) performance status, baseline heart rate, respiratory rate, oxygen saturation, serum albumin level, hemoglobin, LOS, spontaneous pleurodesis with catheter removal, reintervention, and complications. The Fisher's exact test and the $t$-test were used to compare counts and means respectively, and the Wilcoxon rank sum test was used to compare LOS. A P value of 0.05 or less was considered to be the statistical significance threshold.

\section{Results}

We identified 27 patients with a median age of 59.0 years (range, 30.0-92.0 years), 17 (63\%) of which were women (Table 1). Median respiratory rate was 24 breaths/min (range, 18-40 breaths/min); median heart rate 106 beats/min (range, 59-138 beats/min); median oxygen saturation was 96\% (range, 88-99\%); ECOG performance status was 3-4 in $24(89 \%)$ patients; mean serum albumin was $2.49 \mathrm{~g} / \mathrm{dL}$ [standard deviation (SD), $0.48 \mathrm{~g} / \mathrm{dL}$ ], mean hemoglobin was $10.81 \mathrm{mg} / \mathrm{dL}(\mathrm{SD}, 2.39 \mathrm{mg} / \mathrm{dL}$ ) (Table 1). The primary malignancy was lung cancer in $9(33 \%)$ patients, breast cancer in $8(30 \%)$ patients, gastrointestinal cancers in $4(15 \%)$ patients, hematological malignancies in $2(7 \%)$ patients, gynecological cancers in $2(7 \%)$, prostate cancer in $1(4 \%)$ and melanoma in $1(4 \%)$.

The group that received the Aspira group (AG) consisted of $18(67 \%)$ patients and the PleurX group (PG) consisted of $9(33 \%)$ patients. Both groups were similar in age (Table 1), sex, body mass index (BMI), oxygen saturation, hemoglobin and serum albumin on admission.

Spontaneous pleurodesis and IPC removal were achieved in $4(22 \%)$ patients in the AG, in comparison to $3(33 \%)$ in the PG (seven patients overall, 26\%). Additional pleural procedures (catheter removal and reinsertion, pleurodesis, video-assisted thoracoscopy) were required in $6(33 \%)$ patients in the AG and 2 (22\%) patients in the PG.

Median LOS was 9 days (range, 2-38 days) for patients 
Table 1 Baseline demographic data for patients with malignant pleural effusions in the Aspira and PleurX group

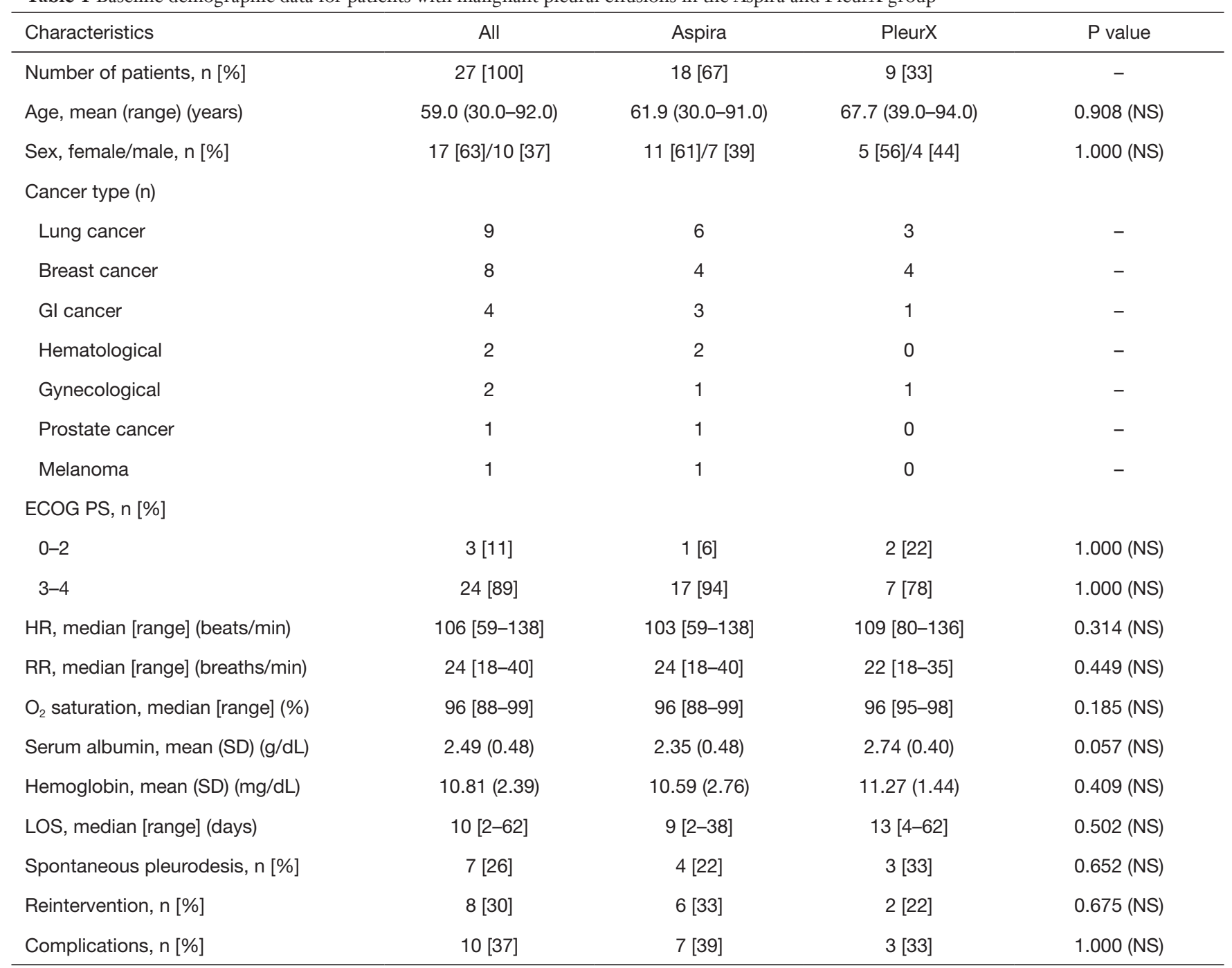

ECOG, Eastern Cooperative Oncology Group; LOS, length of stay; NS, not significant; PS, performance status; SD, standard deviation; $H R$, heart rate; $R R$, respiratory rate.

in the AG, as compared to 13 days (range, 4-62 days) in those in the PG (overall median LOS was 10 days; range, 2-62 days). The rate of catheter-related complications (pain, obstruction, loculations, infection, hemorrhage) was 39\% (seven patients) in the AG and 33\% (three patients) in the PG, (overall ten patients, 37\%). No death was attributed to these events. Intergroup differences were not statistically significant (Table 1). No catheter tract metastasis was observed.

\section{Discussion}

MPE can cause significant respiratory distress and adversely affect the quality of life of the patients, and the best approach to its management remains controversial. As mentioned, that presence of MPE itself determines that the mean survival period of the patient ranges from 6 months to 1 year, depending on the type of cancer (2). Consequently, a desirable approach would combine symptom palliation with limited LOS in the hospital. Treatment options include observation, implantation of an IPC, chemical pleurodesis with a sclerosing agent such as doxycycline, talc powder and bleomycin; and decortication depending on the patient's functional status, type of cancer, and co-morbidities $(5-7,11)$. Observation can be chosen for patients with smaller pleural effusion without any significant respiratory distress or 
if patient has significant other co-morbidities and insertion of tunneled IPC may not result is symptom palliation (12). Chemical pleurodesis includes insertion of the sclerosing agent (talc, bleomycin and doxycycline) between the visceral and pleural space causing an inflammatory reaction, while surgical pleurodesis includes removal of the restrictive fibrinous layer overlying the lung. Both procedures can subject the patient to longer hospital stays and perioperative complications. Talc pleurodesis can also cause pneumonitis, empyema and acute respiratory distress syndrome (ARDS) (11). Insertion of a tunneled IPC involves placement of either PleurX or Aspira catheter into the pleural space. It can be done bedside using the Seldinger technique, it does not require administration of general anesthesia, and the pleural fluid can be collected in the vacuum bottles in PleurX catheter while in Aspira catheter system the fluid is collecting in the bottles with the help of a manual pump attached to the catheter. IPC has very low procedure related morbidity and mortality as compared to surgical procedure like VATS (2). One further advantage is that the IPC can be inserted in an outpatient setting, thereby decreasing utilization of resources (12). IPC can be used for symptom palliation, recurrence and improving the functional status of the patient. It also serves as an ideal option for patients who cannot tolerate pleurodesis or decortication due to shorter life expectancy or increased fragility. IPC has seen to be equally efficacious in symptom control, and spontaneous pleurodesis related to MPE as compared to other procedures $(13,14)$. Warren et al. also found that IPC provides significant palliation, with low complication rates in patients with trapped lung, where other treatment options are doubtful to succeed (14). In our study, both Aspira and PleurX achieved similar outcomes and safety profile. There were no events of catheter tract metastasis, and complication rates were similar in both groups. Reported complications of IPCs are infection at the insertion site, pain and discomfort, malfunctioning of the catheter, catheter tract metastasis, and psychological stress associated with the chest tube and its management.

\section{Limitations}

Our study is limited by the retrospective design, and the small sample size.

\section{Conclusions}

To the best of our knowledge, this is the first study to compare two different types of tunneled IPCs. The findings of our study provide an initial assessment on the efficacy and safety profile of both catheters and did not reveal any evidence to support the use of one type of catheter over the other. Furthermore, they confirm that IPCs are an efficient option for symptom palliation in patients with terminal cancer. Prospective studies with a larger number of patients are required to compare the efficacy and safety profiles of the Aspira and PleurX IPCs.

\section{Acknowledgements}

Funding: This project was partly funded by the Foundation of Yuma Regional Medical Center on behalf of the Richard Michael McDaniel Endowment Fund for Cancer Care.

\section{Footnote}

Conflicts of Interest: The authors have no conflicts of interest to declare.

Ethical Statement: The study was approved by the Institutional Review Board of Maimonides Medical Center (No. 2015-05-06-MMC).

\section{References}

1. Roberts ME, Neville E, Berrisford RG, et al. Management of a malignant pleural effusion: British Thoracic Society Pleural Disease Guideline 2010. Thorax 2010;65 Suppl 2:ii32-40.

2. American Thoracic Society. Management of malignant pleural effusions. Am J Respir Crit Care Med 2000;162:1987-2001.

3. Antony VB, Loddenkemper R, Astoul P, et al. Management of malignant pleural effusions. Eur Respir J 2001;18:402-19.

4. Sahn SA. Pleural diseases related to metastatic malignancies. Eur Respir J 1997;10:1907-13.

5. Myers R, Michaud G. Tunneled pleural catheters: an update for 2013. Clin Chest Med 2013;34:73-80.

6. Putnam JB Jr, Light RW, Rodriguez RM, et al. A randomized comparison of indwelling pleural catheter and doxycycline pleurodesis in the management of malignant pleural effusions. Cancer 1999;86:1992-9.

7. Rehse DH, Aye RW, Florence MG. Respiratory failure following talc pleurodesis. Am J Surg 1999;177:437-40.

8. Adams J, Auger J, Schiff D. Outcome of indwelling 
tunneled PleurX(R) catheter placement in pediatric and young adult patients with malignant effusions. Pediatr Blood Cancer 2014;61:1118-20.

9. Demmy TL, Gu L, Burkhalter JE, et al. Optimal management of malignant pleural effusions (results of CALGB 30102). J Natl Compr Canc Netw 2012;10:975-82.

10. Fortin M, Tremblay A. Pleural controversies: indwelling pleural catheter vs. pleurodesis for malignant pleural effusions. J Thorac Dis 2015;7:1052-7.

11. Bell D, Wright G. A retrospective review of the palliative surgical management of malignant pleural effusions. BMJ

Cite this article as: Gupta SS, Floudas CS, Chandra AB. A comparison between two types of indwelling pleural catheters for management of malignant pleural effusions. J Thorac Dis 2018;10(5):2976-2980. doi: 10.21037/jtd.2018.05.57
Support Palliat Care 2014;4:161-6.

12. Chen H, Brahmer J. Management of malignant pleural effusion. Curr Oncol Rep 2008;10:287-93.

13. Warren WH, Kalimi R, Khodadadian LM, et al. Management of malignant pleural effusions using the Pleur(x) catheter. Ann Thorac Surg 2008;85:1049-55.

14. Warren WH, Kim AW, Liptay MJ. Identification of clinical factors predicting Pleurx catheter removal in patients treated for malignant pleural effusion. Eur J Cardiothorac Surg 2008;33:89-94. 